\title{
Crianza, educación socioemocional y práctica educativa en la perspectiva de agentes educativos de un jardín infantil del programa Buen Comienzo ${ }^{1}$
}

Parenting, socio-emotional education and educational practice from the perspective of educational agents of a kindergarten of the Buen Comienzo program

\author{
Paola Andrea Mesa Villa* \\ (iD https://orcid.org/0000-0002-5427-7721 \\ María Alexandra Rendón Uribe**
}

(D) https://orcid.org/0000-0002-1062-6125

Tipo de Artículo: Informes de Investigación y ensayos inéditos

Doi: 10.17533/udea.unipluri.20.2.015

Mesa Villa, P. A., y Rendón Uribe, M. A. (2020). Crianza, educación socioemocional y práctica educativa en la perspectiva de agentes educativos de un jardín infantil del programa Buen Comienzo. Uni-Pluriversidad, 20(2), e20202015. doi: 10.17533/udea. unipluri.20.2.015

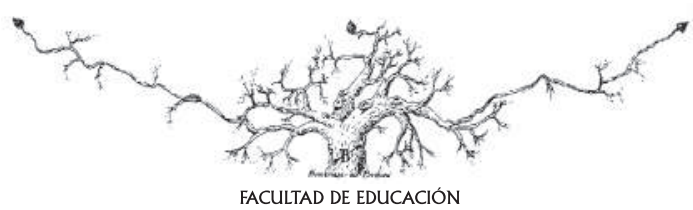

Recibido: 2020-03-26 • Aprobado: 2020-12-22

* Psicóloga U. de San Buenaventura - Medellín y Magíster en Estudios en Infancias de la Universidad de Antioquia en convenio con la UPN. Profesora de cátedra de la Facultad de Educación de la Universidad de Antioquia y Uniminuto. https://orcid.org/0000-0002-5427-7721, paolamesa@gmail.com, https://scholar.google.com/citations?hl=es\&user=7bnmilQAAAAJ

Email: pandrea.mesa@udea.edu.co,

** Licenciada en Educación Preescolar de la U de A, Especialista en Desarrollo del Pensamiento y la creatividad de la U de A, Doctora en Ciencias Pedagógicas del ICCP. Profesora de planta de la Facultad de Educación de la Universidad de Antioquia. Coordinadora del grupo de Investigación Educación, Lenguaje y cognición. https://orcid.org/00000002-1062-6125, https://scholar.google.com/citations?user=FrGU8soAAAAJ\&hl=es

Email: maria.rendon@udea.edu.co 


\section{Resumen}

El artículo presenta los resultados de una investigación desarrollada en una sala cuna de un jardín infantil del programa Buen Comienzo de la ciudad de Medellín. En la investigación se analizó cómo se presentan la crianza y la educación socioemocional en las prácticas de las agentes educativas. En el marco teórico y conceptual se considera al ser humano como un individuo en continuo desarrollo que pertenece a sistemas sociales en constante interacción. Se trata de una etnografía educativa que se inscribe en el paradigma cualitativo, en la que se emplean las siguientes técnicas para la recolección de información: observación participante con registro en diario de campo y una guía de observación compuesta por dimensiones que describen prácticas educativas. Otras técnicas utilizadas fueron un cuestionario escrito y una entrevista semiestructurada, articuladas con la guía de observación, el análisis documental y el grupo de discusión. En el estudio participaron cinco agentes educativas docentes y auxiliares en primera infancia. Los hallazgos develan una estructura de prácticas de crianza extra-familiar que emergen en el rol de las agentes educativas correspondientes a prácticas híbridas entre la educación y la crianza. La educación socioemocional se ubica como un medio y no como un fin para lograr tales prácticas que son identificadas, descritas, analizadas y nombradas. Los resultados de esta investigación constituyen un asunto de interés para programas de educación inicial y profesionales de diferentes disciplinas como educación, psicología, trabajo social, entre otros, porque implica una revisión de los perfiles de formación y del campo laboral de los profesionales que trabajan con la primera infancia.

Palabras clave: crianza, educación socioemocional, práctica educativa, prácticas de crianza extrafamiliar, sala cuna.

\section{Abstract}

The article presents the results of a research carried out in a nursery of a Buen Comienzo program's kindergarten in Medellín city. It analyzed how raising and socio-emotional education are presented in the practices by educational agents. Theoretical and conceptual framework presents an individual approach where the human being is considered in continuous development and belongs to social systems in permanent interaction. This is an educational ethnography defined by a qualitative paradigm with the following information gathering techniques: participant observation with daily records and an observation guide consisting of dimensions to describe educational practices. Other techniques were a written questionnaire and a semi-structured interview, articulated with observation guide, documentary analysis and discussion group. Five educational agents and early childhood assistants participated in the study. Findings reveal a structure of extra-familial raising practices 
that emerge in their role as educational agents. It corresponds to hybrid practices between education and parenting. Socio-emotional education is considered as a means rather than an end to achieve such practices that are identified, described, analyzed, and named. The research results constitute a matter of interest for initial education programs, professionals from different disciplines such as education, psychology, social work, among others, because it implies a review of training profiles and work field of professionals who work with early childhood.

Keywords: raising, socioemotional education, educational practices, extra-familial raising practices, nursery. 


\section{INTRODUCCIÓN}

Hemos sido testigos de los cambios en los roles internos de las familias durante los últimos 60 años; uno de ellos ha sido la creciente incorporación de la mujer al ámbito laboral y, como consecuencia, la necesidad de un apoyo externo al núcleo familiar para la crianza de los hijos. Esto ha conllevado a que sean otros los que participan en el acompañamiento, crecimiento y desarrollo de los niños; entre ellos, los agentes educativos pertenecientes a instituciones de educación inicial $^{2}$. Al respecto, Echenique y Fermín (2011) afirman que el resultado de cambios históricos, socioeconómicos y educativos interrogan sobre la función principal del docente encargado de los primeros años de vida. $\mathrm{Al}$ respecto, los autores señalan que:

El docente se encuentra inmerso en vínculos primarios, es decir, en roles maternales que implican la ejecución de acciones correspondientes a los adultos significativos más cercanos a los niños y niñas, tales como, cambiarles el pañal, acunarlos para dormir o cuando lloran, todo ello desde una mirada profesional y pedagógica (p. 147).

Usualmente, la socialización de la infancia en sus primeros años sucedía exclusivamente en el ámbito familiar, sin embargo, la institucionalización de los niños ${ }^{5}$ se hace cada vez a una edad más temprana. Esto es consecuencia de múltiples transiciones contextuales, sociales, culturales y económicas. Estas variaciones problematizan el lugar de la educación emocional de los niños en ámbitos externos de crianza porque la institucionalización de los niños entre los cero y dos años exige no solo su crianza, sino también un acompañamiento emocional y estrategias de estimulación adecuada por parte de parte de los agentes educativos para promover relaciones de confianza (Vila, 2000; Moreno, 2008; Evans y Myers, 1996; Triana, Ávila y Malagón, 2010; Narodowski y Snaider, 2017). Todos estos elementos se traducen en una suerte de transformación de los patrones tradicionales de crianza y una especie de "hibridación cultural" de los saberes entremezclados de las familias (lo intuitivo) y las instituciones infantiles (lo intencionado). A continuación, se presentan algunos de los conceptos centrales en el estudio.

\section{Lacrianzaylaeducación socioemocional}

La palabra crianza es un derivado de "criatura", que proviene de los verbos, criar, cultivar, crear y producir algo en los seres humanos recién llegados al mundo, mediante manifestaciones de bienvenida y acogida que involucran acciones para responder a sus necesidades, entre ellas: los cuidados iniciales, la protección y el amparo para la supervivencia del niño dado su nivel de dependencia. La crianza es considerada como función de la institución familiar y es una forma de educación basada en lo espontáneo, lo afectivo, lo natural, lo popular y lo intuitivo, surgiendo de saberes que le pertenecen a la educación familiar, diferenciados de los saberes pedagógicos que son propios de los entornos institucionales (Mineducación, 2014; Sacristán, 2003; Soto y Violante, 2008; Arias, 2015, citada en Escobar et al., 2016). Cuidar y proteger es una tarea que hace parte de la organización de una sociedad con el fin de conservarla y transmitirla entre generaciones. Todo ello garantiza la continuidad y el mantenimiento de la 
vida de los recién llegados al mundo. En tal sentido, la responsabilidad de la crianza es labor de toda la sociedad, incluyendo instituciones culturales y actores sociales, por esto es sugerente visibilizar la diversidad de contextos institucionales donde pueden transcurrir las prácticas de crianza de manera integrada con las acciones propias de la educación infantil institucionalizada (Tronto, 1993; Folbre, 2008; Daly y Lewis, 2000, citados en Esquivel, Faur y Jelin, 2012; Soto y Violante, 2008; Escobar et al., 2016).

El agente educativo asume una crianza compartida al acoger a niños menores de dos años en contextos institucionales. Paniagua y Palacios (2005) plantean que muchas de las intervenciones del docente hacia los niños no se diferencian significativamente de lo familiar; sin embargo, se distancian de la intervención familiar caracterizada por lo intuitivo y lo no intencional si sus acciones educativas son de calidad, planeadas, sistemáticas, reflexivas e intencionadas, pedagógicamente pensadas y orientadas a los aspectos de desarrollo del niño. Al articular el modelo planteado por Bronfenbrenner (1987) con el concepto de crianza por fuera del ambiente familiar, se podría decir que el entorno familiar vinculado con el institucional conjuga un mesosistema que procura establecer relaciones bidireccionales. En este caso, cuando los niños cuentan con una edad por debajo de dos años y transitan en ambos entornos, se presentan condiciones para la crianza extrafamiliar; en este sentido, la comunicación entre los entornos familiar e institucional configuran un proceso de intercambio de información y experiencias que permiten referirse a un mesosistema.

Las prácticas de crianza extrafamiliar constituyen una estructura en la que se destacan acciones y acontecimientos que ocurren en la interrelación entre los agentes educativos y los niños. Dichas prácticas entrecruzan procesos pedagógicos con acciones concernientes a la crianza; tal estructura está compuesta de dimensiones y contenidos que determinan las acciones de los agentes educativos docentes en el espacio institucional (García, Loredo y Carranza, 2008).

En este sentido, el agente educativo asume funciones para el acompañamiento del desarrollo socioemocional, es decir, dentro de su práctica educativa, se ocupa de acciones relacionadas con el sostén y contención emocional, la comunicación afectiva, la participación recíproca en expresiones de afecto, la disposición corporal para brindar afecto mediante abrazos, besos, sonrisas, la designación de límites en el entorno, entre otros (Soto y Violante, 2005). Desde una perspectiva fundamentada en los modelos transaccional (Sameroff y Chandler, 1975) y ecológico del desarrollo humano (Bronfenbrenner, 1987), la crianza es una actividad asociada con la interacción afectiva y de progresiva reciprocidad niño-adulto que, en diálogo con el ambiente, operan de manera conjunta para establecer influencias bidireccionales. La interacción mediada por el afecto es un componente de las prácticas de crianza y se revela cuando hay disposición para suplir necesidades, garantizar bienestar en los niños y expresar afecto a través de caricias, besos, abrazos, arrullos, palabras, cuidados básicos y cotidianos, según los ritmos y requerimientos en cada niño (Serna, Hurtado, Mejía, y Zapata, 2000; Sameroff y Chandler, 1975; Bronfenbrenner, 1987; Hernández, 2012).

A partir de estos modelos teóricos, el ambiente es entendido como un entorno interconectado y disponible para que los niños $\mathrm{y}$ agentes educativos desplieguen procesos de interacción afectiva y educación socioemocional en un escenario social dirigido al desarrollo de competencias emocionales, 
evidenciadas en acciones de los niños como identificar, nombrar, conocer, expresar y regular sus emociones con el apoyo de los adultos, así como, empatizar, plantear mecanismos y resolver problemas.

La educación socioemocional exige ambientes llenos de experiencias emocionales y condiciones favorables para su desarrollo y comprensión. Por tanto, al ser un proceso de aprendizaje requiere tiempo y cierto grado de práctica para lograr la apropiación progresiva de pautas de convivencia y relacionamiento social (Barrios-Tao y Peña, 2019; López, 2005; González, de Damas y Vicente, 2020; Shapiro, 2008).

Es importante mencionar que la crianza y la educación tienen una relación vinculante, pero no son equiparables por tres características relevantes: los objetivos que las caracterizan, lo que cada una le permite conocer a los seres humanos y su extensión en el tiempo. Sin embargo, sí es posible y, de hecho, se ponen en diálogo en entornos institucionales como el de una sala cuna.

\section{La práctica educativa y la educación socioemocional}

Según planteamientos de García et al. (2008), la práctica educativa hace referencia a aquello que desde la actuación pedagógica en el entorno institucional ejerce cierta influencia en el niño, por lo tanto, es una actividad dinámica que involucra además procesos de reflexión acerca de la interacción niño-agente educativo. En este sentido, se concentra en tres elementos o dimensiones fundamentales: 1. Pensamiento del agente educativo docente; 2 . Interacción agente educativo docente-niños; y 3. Reflexión acerca de los resultados logrados - registros de la experiencia de las agentes educativas en el contexto de la sala cuna. Esta prácti- ca educativa es el cúmulo de circunstancias delimitadas por el contexto institucional y cuenta con un campo de acción más amplio acerca de lo que acontece por fuera del escenario institucional.

Si se consideran dichas prácticas educativas en relación con la educación socioemocional que se brinda a los niños, es posible estimar algunas dimensiones inspiradas en las propuestas por García et al. (2008) como la personal, la interpersonal, la didáctica y la de empoderamiento y gestión de la crianza aplicadas a las agentes educativas de sala cuna. Estas se describen en la tabla 1.

Lo propuesto hasta ahora y los planteamientos de Vila (2000) abren una perspectiva acerca de los contextos de crianza extrafamiliar que han surgido para promover el desarrollo y educación infantil. La crianza presenta variaciones en sus formas de suceder y para los estudios en infancias es relevante darle un lugar a la discusión acerca de los cambios que implica en el rol de los agentes educativos con conocimientos profesionales y técnicos. De acuerdo con lo presentado hasta ahora, se evidencia una intersección entre lo que serían las prácticas educativas docentes y las prácticas de crianza.

En función de lo anterior, en este artículo se propone responder a la pregunta: ¿Cómo se presentan la crianza y la educación socioemocional en las prácticas de las agentes educativas de una sala cuna del programa Buen Comienzo en la ciudad de Medellín? Para esta investigación, agente educativo hace referencia a la docente orientadora y auxiliares, quienes cuentan con título en licenciatura en pedagogía infantil, licenciatura en educación preescolar, técnica en primera infancia o normalista superior. Los objetivos específicos se orientaron a la descripción de las prácticas educativas que 
realizan las agentes educativas en una sala cuna del programa Buen Comienzo; identificación de elementos propios de la crianza en las prácticas educativas en función de la educación socioemocional de los niños de una sala cuna del programa Buen Comienzo $\mathrm{y}$, finalmente, a develar el papel de las agentes educativas y cómo se asumen en los procesos de crianza y educación socioemocional de los niños de sala cuna.

Tabla 1

Dimensiones de las prácticas educativas

\begin{tabular}{|c|c|c|}
\hline $\begin{array}{c}\text { Dimensión } \\
\text { personal }\end{array}$ & $\begin{array}{c}\text { Dimensión } \\
\text { interpersonal }\end{array}$ & Dimensión didáctica \\
\hline \multicolumn{3}{|c|}{ COMPONENTES } \\
\hline $\begin{array}{l}\text { Autoconciencia } \\
\text { emocional }\end{array}$ & Empatía & $\begin{array}{l}\text { Los recursos físicos y los materiales didácticos como } \\
\text { potenciadores de lo socioemocional (el ambiente o } \\
\text { entorno, los juguetes y los objetos): Autonomía }\end{array}$ \\
\hline $\begin{array}{l}\text { Autovaloración } \\
\text { e integridad } \\
\text { valorativa }\end{array}$ & $\begin{array}{l}\text { Conciencia de } \\
\text { grupo, trabajo } \\
\text { en equipo y } \\
\text { colaboración }\end{array}$ & $\begin{array}{l}\text { La literatura y la expresión teatral (cuentos, } \\
\text { historias, titeres, disfraces, el dramatizado): } \\
\text { Comunicación asertiva } \\
\text { Adaptabilidad emocional }\end{array}$ \\
\hline $\begin{array}{l}\text { Autocontrol } \\
\text { emocional }\end{array}$ & Habilidades sociales & $\begin{array}{l}\text { La lúdica y el juego (canciones, juegos, baile): } \\
\text { Autocontrol emocional, Comunicación asertiva, } \\
\text { Autonomía }\end{array}$ \\
\hline $\begin{array}{l}\text { Autonomía y } \\
\text { autodeterminación }\end{array}$ & Manejo de conflictos & $\begin{array}{l}\text { Despliegue de la motricidad vinculada a lo } \\
\text { socioemocional: Autovaloración, Autonomía, } \\
\text { Autoconocimiento, Comunicación afectiva }\end{array}$ \\
\hline $\begin{array}{l}\text { Adaptabilidad } \\
\text { emocional }\end{array}$ & $\begin{array}{l}\text { Comunicación } \\
\text { afectiva y asertiva }\end{array}$ & $\begin{array}{l}\text { Experiencias provocadoras, retos, oportunidades, } \\
\text { experimentación emocional, sensibilización } \\
\text { o concientización emocional: Autonomía, } \\
\text { Autodeterminación, Habilidades sociales }\end{array}$ \\
\hline \multirow[t]{2}{*}{$\begin{array}{l}\text { Motivación al } \\
\text { logro }\end{array}$} & & $\begin{array}{l}\text { Estímulos para apoyar a los niños: Autonomía, } \\
\text { Motivación al logro }\end{array}$ \\
\hline & & $\begin{array}{l}\text { Integración de los niños y familias al entorno } \\
\text { institucional: Conciencia de grupo, trabajo en equipo } \\
\text { y colaboración }\end{array}$ \\
\hline
\end{tabular}

Categorías deductivas de las prácticas educativas, elaboración propia (2018)

\section{Metodología}

口

Esta es una etnografía educativa inscrita en el paradigma cualitativo. Sus características responden a una forma de investigación sociocultural en el ámbito educativo que implica que el investigador asuma el papel de etnógrafo, quien se fundamenta en la experiencia, la participación y la exploración para la producción de datos descriptivos de un fenómeno en un escenario específico, llámense lenguajes verbales, escritos, corporales 
e interacciones, con el fin de comprenderlo y producir conocimiento. Las participantes fueron cinco agentes educativas del programa Buen Comienzo en la modalidad de entorno institucional de la ciudad de Medellín: una docente orientadora y cuatro auxiliares con conocimientos en primera infancia que atienden una sala cuna con cuarenta niños entre los diez y veinticuatro meses. Para efectos de esta investigación y atendiendo a las consideraciones éticas acerca de la reserva de la identidad, se nombran del 1 al 5: agente educativa uno, agente educativa dos y así sucesivamente. En esta modalidad de atención se ubican las salas cuna, cuyas funciones consisten en brindar atención en un Jardín Infantil que cuenta con infraestructura diseñada y acorde con los requerimientos de los niños durante cinco días a la semana y por ocho horas diarias. La sala cuna que sirvió de escenario cuenta con un espacio amplio e iluminado, dividido en varias partes: lactario, bodega, baños con zona de cambio de pañal y ducha para bebés, además, hay dos zonas con juguetes y objetos distribuidos para los caminadores y otra para los gateadores (Quecedo y Castaño, 2002; Secretaría de Educación de Medellín, 2016).

El trabajo de campo transcurrió en el año 2017 y se emplearon las siguientes técnicas para la recolección de información: observación participante con registro en diario de campo y una guía de observación compuesta por dimensiones que describen prácticas educativas derivadas de competencias socioemocionales en el contexto educativo (García et al., 2008 y otros; Rendón, 2016) que se describen en la Tabla 1.

Otras técnicas que se utilizaron fueron un cuestionario escrito y una entrevista semiestructurada, articuladas con la guía de observación. El análisis documental se empleó para analizar los proyectos de explora- ción y registro de la experiencia escritos por las agentes educativas de enero a junio de 2017. El grupo de discusión cerró el trabajo de campo para profundizar en aquellos datos que se consideraron pertinentes para el logro de los objetivos de esta investigación, a través de una guía con preguntas orientadoras sobre la educación socioemocional, socialización y prácticas de crianza. La entrevista y el cuestionario tuvieron valoración de expertos y una prueba piloto. El consentimiento informado hizo parte de las consideraciones éticas según planteamientos de la Resolución $\mathrm{N}^{\circ} 008430$ de 1993, del 4 de octubre de 1993, del Ministerio de Salud de Colombia que contempla protección de los derechos de todos los sujetos participantes.

Durante el análisis de los datos, se consideraron varios momentos: reflexión analítica, selección, reducción y organización, codificación abierta, categorización y codificación axial. Las categorías fueron agrupadas y reagrupadas a modo de una red de datos (Strauss y Corbin, 1990, citados en Rodríguez, 2003 y Álvarez, 2011). Además, el proceso de análisis de los datos es un momento creativo de autodiálogos y autocríticas permanentes en concordancia con el marco referencial de teorías y conceptos que enmarca el campo de conocimiento académico. Este momento de la investigación implicó trascender la descripción hacia un proceso de análisis para interpretar el fenómeno estudiado (Schettini y Cortazzo, 2015).

Los procedimientos previos a la escritura de los resultados y conclusiones necesitaron verificación de autenticidad y credibilidad de los datos, a través de la validación con la triangulación de métodos, confrontando los datos recolectados de la observación participante, cuestionario, entrevistas semiestructuradas, análisis documental y grupo de discusión. A pesar de contar con 
la observación como técnica por excelencia, la etnografía no es incompatible con la estrategia de triangulación, ni mucho menos con otras técnicas de recolección de datos. (Hernández Sampieri, Fernández y Baptista, 2014; Álvarez, 2011).

\section{Resultados}

En esta sección se presentan los resultados organizados en tres apartados: el lugar de la crianza en la sala cuna, la educación socioemocional desde la práctica educativa y sus dimensiones e hibridación de prácticas y contenidos de la crianza extrafamiliar.

\section{El lugar de la crianza en la sala cuna}

Al interrogar a las agentes educativas sobre el concepto de crianza se encontraron elementos concernientes con acciones afectivas, promoción del desarrollo moral y emocional, y la transmisión de valores a los niños.

La discusión sobre quién se empodera de la crianza se dio en planos diferentes porque algunas agentes educativas la ubican únicamente en el entorno familiar; mientras tanto, otras reconocen que la crianza se da en ambos entornos (familiar e institucional), tanto en su discurso como en sus prácticas. En las respuestas de las agentes educativas sobre su papel en los procesos de crianza se da cuenta de la incidencia que tiene el entorno familiar en sus intervenciones, debido a las permanentes demandas de las familias frente a lo que requieren los niños cotidianamente, y muestran variadas posturas sobre su papel en la crianza de los niños de sala cuna. Esto se evidenció en el grupo de discusión como se indica en los siguientes apartados.

\section{La crianza como acción de proteger.}

Algunas agentes educativas ponen de manifiesto funciones protectoras al identificar que los niños necesitan de más cariño por la temprana edad cronológica y, debido a que aún no tienen un lenguaje hablado estructurado, los sitúan en un lugar frágil y carente en el que requieren de más atención y protección. Las acciones para proteger se encuentran asociadas con cuidar y asumirlos como hijos.

En la entrevista semiestructurada, la agente educativa dos alude al apoyo y a la posibilidad de ayudarlos porque los concibe como niños en estado de desprotección y desamparo por estar por fuera de su entorno familiar. Ella emplea una expresión que llama la atención al momento del análisis, "la crianza de nosotros", esto lleva a pensar en una crianza al modo de ellas, es decir, otra forma de generar crianza, distinta a la que se vive en el entorno familiar y cambiante en dos sentidos: por el entorno de ocurrencia y por los actores que la ejecutan.

\section{La figura materna de las agentes educativas en la sala cuna.}

La maternidad asumida con los hijos biológicos es referente en su papel en la sala cuna. Así lo deja saber la agente educativa tres, quien fue enfática en manifestar que ejercer la maternidad con su hija biológica le sirve como punto de apoyo en su rol en sala cuna. Los niños de sala cuna, a pesar de no ser hijos biológicos de las agentes educativas, requieren de un alto nivel de responsabilidad con ellos. Este es un asunto que representa y equipara, de alguna forma, la responsabilidad de ser padres, además, es un acto de corresponsabilidad de la sociedad y el Estado con la protección de los niños. 
En estas consideraciones están presentes los planteamientos de Bravo Soto (2018) cuando, en las entrevistas a las madres que hicieron parte de ese estudio, estas consideran que, en el jardín infantil, las agentes educativas se ocupan de ciertas labores maternales referidas a los horarios del bebé, sus hábitos de sueño, ingesta de alimentos y las mudas.

Si bien estos razonamientos guardan cierta lógica, es preciso tener presentes unos parámetros laborales estructurados en cuanto a las funciones de la agente educativa docente o auxiliar de sala cuna de modo que no dé pie a distorsiones del rol.

\section{La crianza y los vínculos afectivos, significativos y preferenciales}

En la línea de lo que se viene mencionando emergió la categoría de acompañamiento afectivo para el bienestar de los niños. Esta puede asociarse a la vinculación afectiva y se traduce en la disponibilidad para el establecimiento de vínculos, no solo de carácter afectivo sino también significativo y preferencial (Hernández, 2012). Esta interpretación se da porque al encontrarse en ellas disposición corporal y afectiva en la interacción con los niños, también se identificaron procesos que dan cuenta de la construcción e instalación de vínculos afectivos significativos en la sala cuna estudiada. Esta es una de las respuestas de dicho entorno a los cambios históricos, socioeconómicos y educativos descritos en el planteamiento del problema de esta investigación; en la sala cuna se forma un tejido entre el papel de criar y educar, hallándose en el medio la vinculación afectiva.

Al respecto, Echenique y Fermín (2011) han interrogado acerca de la función principal del docente con niños institucionaliza- dos durante sus dos primeros años de vida. En esta sala cuna se da una vinculación afectiva porque las agentes educativas asumen la atención de los niños menores de dos años según sus características afectivas y emocionales. Otro elemento que se suma es el lugar que les otorgan los niños a las agentes educativas cuando las nombran "ma" o "tías", lo cual tiene consecuencias en las formas de relación y en el lugar que asumen. Este es un factor que influye en el establecimiento de tejidos y apegos.

Durante esta investigación se reafirma lo planteado por Soto y Violante (2008) acerca de las características del entorno para que surja el desarrollo de vínculos afectivos y significativos con niños menores de dos años, esto es, condiciones de acogida, cuidados y contacto frecuente por medio de acciones propias de la cotidianidad, como acunar, hablar con afecto, arrullar, cargar. Estas son manifestaciones ligadas a la labor materna. También es posible afirmar que se identificaron elementos que hacen pensar en vínculos afectivos preferenciales, caracterizados por una atención con tratos exclusivos, es decir, la mayoría de las agentes educativas generan vínculos muy cercanos con aquellos niños que tienen cierta semejanza con sus hijos biológicos. Al respecto, UNICEF (2012) confirma lo hallado en esta investigación al hacer referencia a que a veces se gestan unos vínculos más fuertes, significativos y preferenciales que otros.

\section{Lugar para cuidar junto con las familias}

El dilema entre cuidar y educar está circulando permanentemente en el papel de las agentes educativas de la sala cuna porque encuentran factores externos influyentes en sus prácticas. Acá, las familias juegan 
un papel en la percepción que tienen de las agentes educativas como cuidadoras de sus hijos. Es claro que las familias depositan su confianza en las agentes educativas al dejar a sus hijos a su cuidado, pero tales percepciones generan cierta reducción o restricción del rol de las agentes educativas. A pesar de que el programa Buen Comienzo tiene establecidos unos lineamientos con intenciones pedagógicas, tienen lugar en el ambiente educativo las funciones de asistir y cuidar. La agente educativa cuatro manifiesta reclamos dirigidos a las familias de los niños de sala cuna ya que sus gestiones pedagógicas quedan invisibilizadas porque los intereses o expectativas de las familias se concentran en acciones dirigidas al cuidado de los niños en las que se dejan de lado experiencias pedagógicas.

Según estas manifestaciones, las familias conocen poco sobre las funciones de las agentes educativas. Contrario a ello, la agente educativa cinco, en el grupo de discusión, resalta la actitud de una de las mamás quien, al inicio del calendario escolar, se interesaba por conocer el desarrollo de actividades pedagógicas durante el día, sin embargo, ese interés fue diluyéndose. En general, se puede decir que las expectativas de las agentes educativas docentes y auxiliares sobre su rol distan de las que albergan las familias.

Las familias interponen en el papel de las agentes educativas cierta presión, por ejemplo, la agente educativa cinco afirmaba en el grupo de discusión: "trabajamos como para cumplirles a ellos". Ella estaría insinuando la ejecución de algunas acciones con los niños por la presión familiar para responder o satisfacer las demandas manifestadas por las familias.

Las experiencias cotidianas de atención, asistencia y cuidado por parte de las agentes educativas hacia los niños influyen en el nacimiento y estructuración de vinculaciones afectivas significativas; es un acto de tejer vínculos que fomenta su desarrollo socioemocional. La instalación de estos vínculos afectivos entendidos como experiencias afectivas significativas, como abrazos, besos y caricias, indican una acogida social y afectiva, y cuidados desde el plano físico/ biológico para su supervivencia, lo cual ratifica la crianza por fuera del entorno familiar. En el caso de esta sala cuna, las agentes educativas atienden estos requerimientos de los niños con una intensidad mayor o menor según cada caso, lo que genera la estructuración de vínculos afectivos y es una evidencia del enlazamiento de las prácticas educativas y de crianza mediadas en ambos casos por expresiones afectivas y respuestas sensibles hacia los niños. Se concluye que algunos elementos característicos de las prácticas de crianza se evidenciaron en las prácticas de estas agentes educativas: manifestaciones desde el amor, el cariño, la incondicionalidad, la sensibilidad por el estado de ánimo y expresiones emocionales de cada niño.

\section{La Educación socioemocional desde la práctica educativa y sus dimensiones}

Desde el punto de vista de las agentes educativas, la gestión de la educación socioemocional nace en el entorno familiar, se refleja en el institucional y es complementada cuando se generan acciones para abordar o reforzar, en algunos casos, lo que los niños traen de sus hogares. Para que la gestión de la educación socioemocional tenga lugar y continuidad en el entorno institucional, las agentes educativas destacan la importancia de mantener una mirada crítica y objetiva del entorno familiar y la influencia en el niño, es decir, ir al fondo de cómo es su familia para comprender sus comportamientos 
por medio de la observación detallada, profunda y comprensiva para analizar al niño y la influencia de su entorno familiar.

Los aportes entregados por Serna et al. (2000) dan sentido a la necesidad de acompañamiento emocional de los niños institucionalizados y menores de dos años, quienes no solo necesitan ser cuidados, merecen estimulación y relaciones basadas en confianza y afecto. Es necesario dar relevancia a las necesidades emocionales sobre las necesidades físicas, alcanzando interacción afectiva y construyendo relaciones de apego.

Durante el grupo de discusión, la agente educativa cinco afirma que "la primera profesora de los niños se vuelve referente y es una persona significativa". Lo anterior se puede enlazar con las características de la socialización primaria como un proceso que "implica el desarrollo de la identidad individual y social, un yo propio y un vínculo social" (Moreno 2008, p. 13), que funda bases para las siguientes experiencias de vida. Esta afirmación abre la discusión acerca de si se extienden o no procesos de socialización primaria en contextos por fuera de la familia durante estos primeros meses de vida. Cabe preguntarse si lo que diferencia a la socialización primaria con la secundaria está supeditado exclusivamente al entorno en el que suceden las primeras interacciones, la edad de los niños o los actores involucrados. Al respecto Moreno (2008) plantea que:

Los sistemas psicológicos tradicionales han atribuido un papel totalizante a la crianza, planteando una función determinista entre ésta y la socialización, aunado a un papel receptivo-pasivo del individuo socializado. Posteriores desarrollos han permeado esta óptica, planteando importantes cambios en su comprensión..., ubicando la socialización en el marco de interacciones complejas. (p. 9)
Lo anterior implica que, aunque se considera con frecuencia a los padres como los responsables y agentes más influyentes en los procesos de socialización, es claro que en las trayectorias del desarrollo individual y familiar se van recibiendo influencias de otros agentes de socialización y, por tanto, el resultado de este proceso se ve afectado. Al preguntarles por el lugar que tiene la educación socioemocional en sus prácticas educativas, varias consideran este aspecto como fundamental. Una de las pistas que usan para identificar problemáticas de orden socioemocional en los niños son sus reacciones emocionales, lo cual las lleva a indagar las razones o causas de sus comportamientos.

Pese a lo anterior, los resultados de esta investigación muestran que la educación socioemocional brindada por las agentes educativas es un medio y no un fin porque no se identifica la estructuración intencionada en la planeación. Investigaciones realizadas por Merino (2015) y Ramírez, Ferrando y Sainz (2015) afirman que la educación socioemocional no hace parte del currículo en programas de primera infancia, ni del contenido de las planeaciones. Sin embargo, al observar las prácticas realizadas, sí se identifican acciones hacia la educación socioemocional con un carácter espontáneo e intuitivo. Algunas prácticas educativas identificadas que favorecen la educación socioemocional de los niños son:

Desde la dimensión personal de las prácticas educativas, el componente de autoconciencia emocional de las agentes, pues en la medida en que ellas reconocen sus emociones pueden favorecer la asimilación e incorporación de herramientas emocionales adecuadas en los niños; este componente junto con las manifestaciones físicas de afecto y amor favorecen el establecimiento 
de vínculos afectivos que a su vez promueven el desarrollo de los niños.

En la dimensión interpersonal y a partir de los registros realizados (guía de observación), es notable la observación intencionada y el acompañamiento afectivo que hacen las agentes educativas para el bienestar de los niños con la finalidad de alcanzar su comprensión. Mediante esa observación, las agentes llegan a conocer las preferencias y desagrados de cada niño, es decir, sus características individuales. Adicionalmente, las respuestas afectivas de las agentes surgen como resultado de la identificación del estado emocional de cada niño $\mathrm{y}$, como respuesta, en algunos casos de desagrado, las agentes acuden a la palabra para brindar tranquilidad y confianza a los niños.

Este tipo de prácticas que se hayan vinculadas con la identificación de estados emocionales son reconocidas por Hernández (2012), quien propone que una de las funciones de las agentes educativas es el reconocimiento de las emociones de los niños mediante una actitud de escucha y de estar alerta. También hacen parte de esta dimensión interpersonal el trato cálido y acogedor, intencionado, y espontáneo, los intercambios sociales y la promoción de valores para la incorporación social de los niños y el desarrollo de sentimientos sociales en ellos (Piaget, 1981, citado en Wadsworth, 1991).

En la dimensión interpersonal de las prácticas también cobra sentido el componente de habilidades sociales de las agentes educativas, desde allí, se fomenta la socialización (Soto y Violante, 2005). De acuerdo con elementos aportados por Moreno (2008), la socialización primaria permite a los niños hacer parte de un grupo social, formar su propio mundo y, del mismo modo, desplegar identidad social y personal, desarrollo moral y adquirir un lenguaje. Los contenidos de la socialización primaria están referidos a brindar a los niños bases sociales y culturales en términos de normas, valores y comportamientos dados en un entorno con una fuerte carga emocional a raíz de los vínculos afectivos; son bases que se vuelven referentes para la interacción actual y futura de los niños. Además, su interacción con compañeros de sala y con las agentes educativas les ayuda en la comprensión de las formas de funcionamiento de las relaciones humanas. La intervención directa de las agentes para sostener y regular las emociones de los niños y el acompañamiento en el aprendizaje de comportamientos socialmente aceptados hacen parte también de procesos de socialización primaria (Soto y Violante, 2005; Rubio, citado en Linde, 2010; Kohlberg, citado en Linde, 2010).

Desde la dimensión didáctica fueron significativas las estrategias basadas en la lúdica y en el juego orientadas a la regulación y promoción de la expresión emocional, como los juegos en el espejo y las canciones infantiles. En estas actividades, los niños van conociendo y comprendiendo las formas de funcionamiento de las interacciones humanas, los comportamientos socialmente aceptados que los fundamentan social y culturalmente, por ejemplo, apropiar elementos socioculturales, como el uso de cubiertos en el comedor mediante canciones infantiles, música y coreografías. Además, para promover la expresión y regulación emocional usan la literatura, expresión teatral, dramatizaciones, cuentos, historias, música y baile (Rubio, citado en Linde, 2010; Kohlberg, citado en Linde, 2010).

Los hallazgos generales de la dimensión didáctica, en la que se dan prácticas alrededor de la lúdica, el juego, la literatura, el cuerpo $\mathrm{y}$ todos aquellos gestos de estimulación, posibilitadores de exploración, expresión y re- 
gulación de las emociones de los niños en el entorno institucional, demuestran las gestiones desarrolladas por las agentes educativas para la educación socioemocional de los niños en las que, de forma relevante, velan por el desarrollo de la autonomía de los niños. En el marco de estas dimensiones de la práctica se hallaron comportamientos y acciones enfocados a la educación socioemocional y al bienestar psicosocial de los niños. Como lo han expuesto Evans y Myers (1996) y Hernández (2012), son contenidos que tienen un enfoque socioafectivo y emocional al procurar estabilidad en los niños.

\section{Hibridación de prácticas y contenidos de la crianza extrafamiliar que se abordan en la sala cuna}

En el grupo de discusión, las agentes educativas elaboraron conclusiones en las que afirmaron que las prácticas educativas y de crianza se enlazan en las acciones y actividades cotidianas que pueden verse en la sala cuna. Esto indica un reconocimiento de la hibridación de prácticas en su quehacer, debido a que hay algo enlazado o entretejido entre las prácticas de crianza en sala cuna y las prácticas educativas. La agente educativa cinco insinúa que se podría asignar un "nombre científico" que dé cuenta de las relaciones entre ambas prácticas en su labor en sala cuna. Por su parte, la agente educativa dos asocia las prácticas de crianza con asistencia y cuidado, y las prácticas educativas con enseñanza, aprendizaje y orientación en valores.

La conexión identificada de la educación socioemocional con las prácticas educativas y de crianza se da a partir de elementos hallados en las agentes educativas que fueron tener apertura y disposición emocio- nal. Estos son aspectos influyentes en las maneras de tratar a los niños y comunicar las emociones. Algunas manifestaciones y contenidos de la crianza extra-familiar que lograron identificarse son:

\section{Normas básicas de comportamiento social}

Las familias suelen acompañar a los niños en sus primeros acercamientos a normas básicas de comportamiento y las enseñanzas de las agentes educativas en la sala cuna también sirven de fundamento para el comportamiento social de los niños, a través de acciones repetitivas que se convierten en rutinas: dar las gracias, saludar, despedirse, saber qué hacer para convivir con pares y adultos. Para la agente educativa tres es relevante el factor tiempo al referirse a la amplia permanencia de cada niño en la sala cuna, circunstancia que es propicia para que ellas aporten a estos contenidos de la crianza. Moreno (2008) afirma que tanto la familia de origen como la institución educativa dan soporte a la socialización y dan cuenta de las normas compartidas social y culturalmente. De eso se trata precisamente la socialización, "como un proceso a través del cual, determinada sociedad u orden social logra pervivir y reproducirse, trasmitiendo a los nuevos miembros aquellas normas y principios necesarios para la continuidad del sistema" (p 13).

\section{Logros socio afectivos significativos}

Los niños establecen vinculaciones afectivas significativas con alguna de las agentes educativas y viceversa, esto proporciona seguridad emocional y sirve de soporte para la estructuración y mantenimiento de relaciones interpersonales constituidas por el afecto, el amor y el cariño. En esta sala 
cuna, el afecto suele ser el mediador de las interacciones para que el trato a los niños sea cálido y acorde con sus necesidades. Las mismas reacciones emocionales de los niños pueden demostrar que las agentes educativas les representan una vinculación afectiva importante.

Otro elemento que logra ponerse de manifiesto son los grandes hitos del desarrollo durante los primeros años de vida que se evidencian en el entorno institucional, aspecto que representa para el ser humano avances valiosos y trascendentales. Acontecimientos tan importantes como las primeras palabras y los primeros pasos de cada niño, que antes eran festejados por los padres y las familias, ahora son experiencias que se viven y celebran en el entorno institucional y son muy significativas, tanto para los niños como para las agentes educativas de la sala cuna.

De acuerdo con Martínez y García (2012), esta complejidad en las relaciones interpersonales y afectivas exige nuevas comprensiones sobre la forma en la que los agentes educativos y los niños se influyen recíprocamente a lo largo del tiempo, de manera que se logren intervenciones más asertivas que ayuden a las familias que enfrentan dificultades o a los niños en riesgo y promuevan su desarrollo.

\section{La confianza e independencia/ autonomía}

Los niños en su condición de recién llegados al mundo son apoyados por las agentes educativas quienes fomentan su confianza e impulsan su independencia/autonomía. La confianza y autonomía son los principales logros en los niños que son favorecidos desde las prácticas e interacciones con las agentes educativas. Como lo confirma Eri- kson (1963, citado en Shaffer, 2002), las condiciones apropiadas para que los niños logren confianza básica se concentran en la interacción cotidiana; esta interacción favorece la atención de necesidades emocionales básicas de los niños. Al respecto, Soto y Violante (2005) afirman que son funciones para el sostenimiento y contención emocional de los niños de sala cuna establecer con ellos comunicación afectiva, disponerse corporalmente para brindarles afecto, entre otros.

En este orden, los niños menores de dos años necesitan contar con adultos sensibles dispuestos a brindar respuestas en un ambiente que inspire tranquilidad. Para potenciar la confianza es necesario un rol basado en la comprensión y la empatía; la agente educativa debe saber qué hacer sin llegar al maltrato.

Los tres elementos de la crianza extrafamiliar que se desarrollaron constituyen un emergente en las prácticas de las agentes educativas y dan cuenta de una intersección de prácticas de crianza y educativas a modo de híbrido que se nombran en esta investigación como prácticas de crianza extrafamiliar. Estas prácticas se han generado como producto de cambios contemporáneos y dan cuenta de las transformaciones que se han desatado con la institucionalización de los niños a temprana edad.

Esta hibridación de prácticas puede ser explicada desde la teoría ecológica del desarrollo humano de Bronfenbrenner (1987). Frente a los constructos de esta teoría, serían sistemas o ambientes incluidos uno dentro del otro donde los niños participan y se establecen comunicaciones entre los miembros que los conforman; cada ambiente es un microsistema que al entrar en interacción conforman un mesosistema, como lo indica la siguiente gráfica: 


\section{Gráfica 1}

Intersección de prácticas educativas y de crianza. Elaboración propia

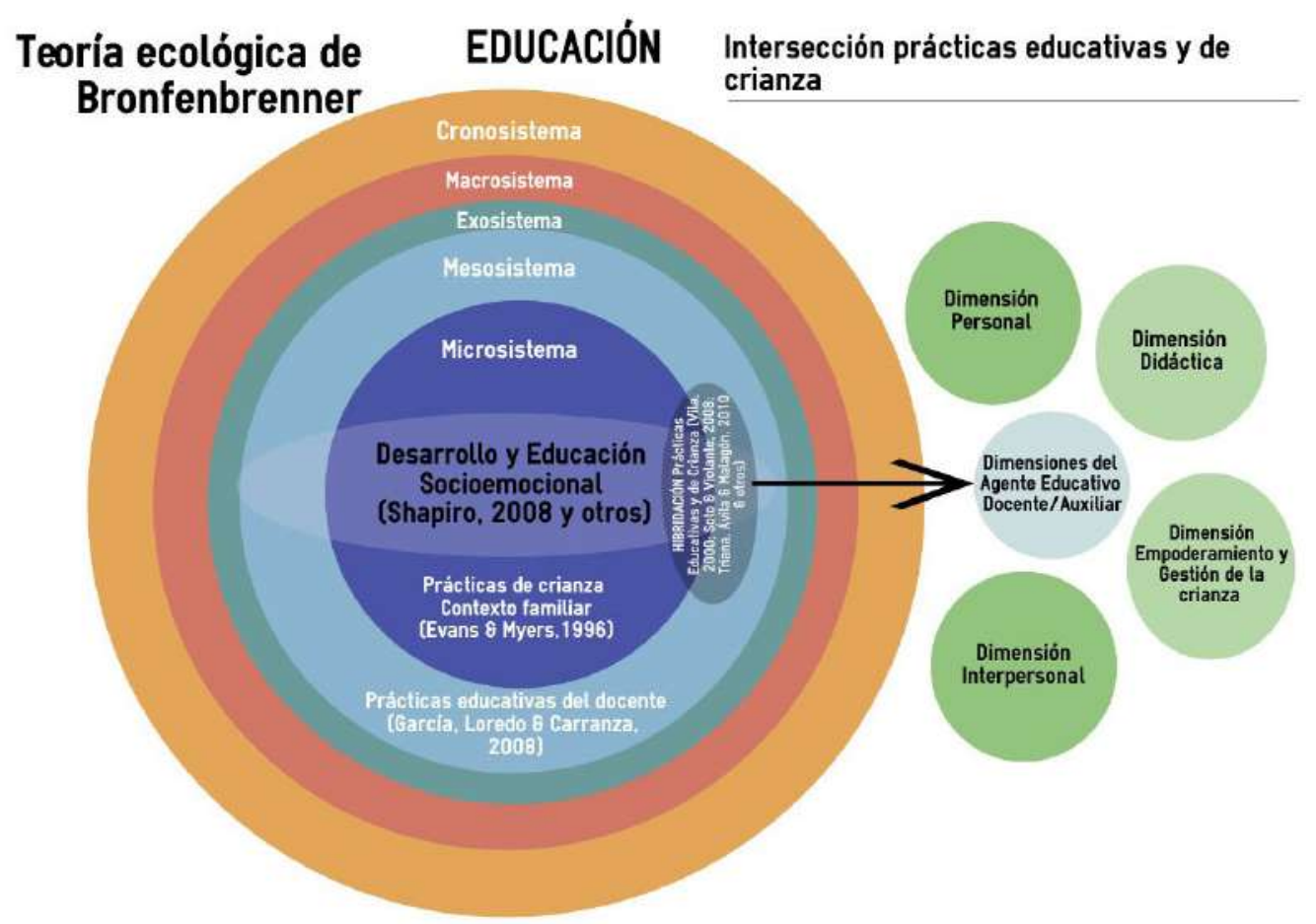

Esta manifestación de hibridación la confirman Triana et al. (2010) quienes proponen que, de acuerdo con los cambios sociofamiliares contemporáneos, hay una transformación de patrones convencionales de crianza, originando hibridación cultural, provocada por la mezcla entre lo que conocen las familias y lo que conocen las instituciones infantiles.

En las relaciones del entorno institucional con el entorno familiar se encontraron dos modos de relacionamiento, por un lado, el preferencial y, por otro lado, el conflictivo. El modo conflictivo fue evidenciado por los frecuentes reclamos y cuestionamientos de las agentes educativas hacia algunas responsabilidades poco asumidas de las familias en torno a los niños.

Reclamar la corresponsabilidad de las familias y de la sociedad para acompañar a los niños, exige establecer canales de inte- racción e integración entre ambos entornos para la colaboración y el trabajo conjunto en función del bienestar de los niños. Esta es una invitación para la reacomodación y reconfiguración de responsabilidades, funciones y roles. Este reto debería ser liderado por el entorno institucional para plantear relaciones más claras entre las funciones de las familias y el perfil laboral de las agentes educativas sin desconocer las transformaciones sociofamiliares y educativas que se vienen dando.

La crianza extrafamiliar implica pensar en un trabajo cooperativo y una mirada flexible de la crianza. Se abre la invitación para que futuras investigaciones se ocupen de profundizar acerca de lo que acontece con la socialización primaria al transitar la crianza entre un entorno y otro y cómo se podrían estudiar los procesos de socialización secundaria en el entorno institucional. 


\section{Conclusiones}

Esta investigación despeja el horizonte de las prácticas de crianza presentes en esta sala cuna. Se halla un ambiente que responde a los requerimientos socioafectivos de los niños menores de dos años, al contar con unas condiciones específicas que desarrollan componentes sustentados en la estructura de prácticas de crianza extrafamiliar en términos de implicaciones de carácter afectivo, vincular y de experiencias exclusivas que no presencian los padres.

Al identificar aquellas prácticas de crianza que realizan las agentes educativas en función de la educación socioemocional de los niños en la sala cuna, se halló el entrecruzamiento o intersección de prácticas de crianza y prácticas educativas. Este es un híbrido que en ciertas obras se denomina crianza extrafamiliar y apoya la construcción de normas básicas de comportamiento social, instauración de vínculos afectivos significativos y fomento de la confianza e independencia/autonomía de los niños. En otras palabras, es posible afirmar que en el contexto de las salas cunas existe la crian$\mathrm{za}$, un entorno distinto al familiar donde son precisamente las agentes educativas (licenciadas, normalistas o técnicas) quienes dan vida a las prácticas de crianza desde su quehacer en este escenario.

Como resultado del análisis del papel de las agentes educativas docente/auxiliar y cómo se asumen en los procesos de crianza y educación socioemocional de los niños de sala cuna, se encontraron varios matices que desvelan su rol. Este transcurre entre la protección, la maternidad y el cuidado; ellas ocupan un lugar relevante para los niños al percibirse como referente y punto de apoyo. El rol de agentes educativas también mues- tra una tensión que se mueve en la disyuntiva entre cuidar y educar. Desde los lineamientos pedagógicos del programa Buen Comienzo y también desde la formación académica, se insta por favorecer la educación, pero es claro que, debido a la naturaleza de la sala cuna y de quienes la habitan, el cuidado también tiene un lugar preponderante Así mismo, su papel se haya influenciado por las presiones de las familias, por lo que su rol como agentes educativas exige ese un cruce entre las prácticas de crianza con las prácticas educativas.

Dicho de otro modo, las prácticas de crianza llegan a un entorno institucional que no es el familiar, allí ocupan un lugar poco convencional para las expectativas profesionales y laborales de las agentes educativas que, a pesar de todas ser madres de familia, cuesta aceptar por fuera del ambiente familiar, dentro de su lugar de trabajo.

Así las cosas, un diálogo entre las prácticas de crianza y las prácticas educativas es recomendable para comprenderlas y asumirlas entrelazadas como una estructura emergente e incorporarlas explícitamente en los proyectos pedagógicos buscando un punto de encuentro con el entorno familiar que ayude en su convergencia.

Esta investigación constata la presencia de prácticas de crianza en el entorno institucional acompañada de procesos de educación socioemocional en el quehacer de las agentes educativas de la sala cuna. Los hallazgos confirman que el lugar que ocupa la crianza y la educación socioemocional se ubica en las prácticas conjugadas y/o entremezcladas en el rol de las agentes educativas, traspasando la frontera del mundo 
íntimo y privado del entorno familiar y el contexto de la sala cuna, ocupando un lugar en medio de la intersección de ambos entornos y constituyendo unas prácticas híbridas y emergentes.

La educación socioemocional es un hilo o conector vinculado a la crianza en la sala cuna y se identifica como el motor principal en sus acciones. A pesar de ubicarse en ese lugar relevante, llama la atención que es una categoría implícita porque es ejecutada, pero no se nombra como tal. Además, no es un proceso sistemáticamente planeado.

La conjunción de prácticas educativas y de crianza se está agregando al papel de agentes educativas para niños de sala cuna, para ello se requiere mayor sinergia entre ambos entornos con el fin de hacer ajustes a las funciones de los actores en cada contexto y, así, establecer fronteras para la reconfiguración de cada entorno de acuerdo con los cambios dados en algunas funciones de las familias en la contemporaneidad. Por tanto, los encuentros de familias liderados por el personal del jardín infantil pueden ser la oportunidad para realizar tales ajustes y para velar de forma conjunta por los derechos fundamentales de los niños de manera que se pueda intervenir situaciones problemáticas bajo un enfoque interdisciplinario para lograr que las familias se empoderen y mantengan una relación favorable con el jardín infantil.

En definitiva, describir y asignarle nombres a lo que viene sucediendo en las prácticas de las agentes educativas es favorable porque les ayuda a entender la confluencia de prácticas que circulan del entorno familiar al institucional. De esta forma, estos nuevos significados pueden influir de forma relevante en las funciones de las agentes educativas y lograr el encuentro de elementos beneficiosos para la atención integral de niños en sala cuna. Así pues, la crianza extendida al entorno institucional debe encontrarse con elementos pedagógicos que la sustenten como una forma de abordar a los niños institucionalizados con una edad inferior a los dos años y debe servir para poner en diálogo las disyuntivas presentes en el rol de las agentes educativas.

\section{Notas}

1. Artículo de investigación. Área de conocimiento: Educación Infantil. Trabajo derivado del trabajo de grado: Crianza, educación socioemocional y práctica educativa en un jardín infantil del programa Buen Comienzo, presentado para optar el título de Magister en Estudios en Infancias, Universidad de Antioquia, Medellín, Colombia.

2. En educación inicial existen variadas figuras de Agentes Educativos (AE). Según el Ministerio de Educación Nacional de Colombia, los agentes educativos son todos aquellos adultos profesionales o no profesionales en educación que satisfacen las necesidades básicas cotidianas de los niños menores de cinco años y están involucrados en la atención integral de los mismos bajo el principio de la corresponsabilidad (Mineducación, 2017). Sus acciones pueden fundamentarse en conocimientos y saberes de diversas fuentes según su rol ante los niños; para este caso se incluyen como AE: padres (adultos con conocimientos, saberes intuitivos y acciones espontáneas); docentes y auxiliares (adultos con conocimientos y saberes académicos, sistematizados, pedagógicos, acciones y situaciones, planeadas e intencionadas). 
3. Este artículo emplea el concepto niños siguiendo principios de la economía del lenguaje. Por tanto, representa a los niños y las niñas para evitar repeticiones que generan dificultades sintácticas y restan fluidez a la redacción y la lectura. (RAE) http://www.rae.es/consultas/los-ciudadanos-y-las-ciudadanas-los-ninos-y-las-ninas\#sthash.dWgcOKdc.dpuf Además se refiere a seres humanos con derechos, protagonistas de su propia vida, recién llegados al mundo, es decir, cubre cronológicamente los primeros 2 años de nacimiento.

\section{REFERENCIAS}

Álvarez, C. (2011). El interés de la etnografía escolar en la investigación educativa. Estudios pedagógicos. 37(2), 267-279. http://www.scielo.cl/pdf/estped/v37n2/art16.pdf

Barrios-Tao, H., y Rodríguez, L. J. P. (2019). Líneas teóricas fundamentales para una educación emocional. Educación y Educadores, 22(3), 487-509. Doi: http://dx.doi.org.ezproxy.uniminuto. edu/10.5294/edu.2019.22.3.8

Bravo Soto, A. (2018). Prácticas de crianza en la sala cuna y en el hogar: un estudio de caso. Educación, 27(53), 7-23. https://dx.doi.org/10.18800/educacion.201802.001

Bronfenbrenner, U. (1987). La ecología del desarrollo humano. Ediciones Paidós Ibérica, S.A.

Echenique, A., y Fermín, M. (2011). Atención pedagógica para la etapa maternal: una revisión del proceso de formación de educadores infantiles. Revista de investigación, 35 (72), 143-165. http:// www.scielo.org.ve/scielo.php?script=sci_arttext\&pid=S1010-29142011000100009

Escobar, J., González, M., y Manco, S. (2016). Mirada pedagógica a la concepción de infancia y prácticas de crianza como alternativa para refundar la educación infantil. Revista Virtual Universidad Católica del Norte, 47, 64-81. http://revistavirtual.ucn.edu.co/index.php/RevistaUCN/ article/view/742/1268

Esquivel, V., Faur, E., y Jelin, E. (Eds.). (2012). Las lógicas del cuidado infantil. Entre las familias, el Estado y el mercado. http://cddhh.ides.org.ar/files/2012/06/Las-1\%C3\%B3 gicas-del-cuidado-infantil.-Entre-las-familias-el-Estado-y-el-mercado.1.pdf\#page $=74$

Evans, J., y Myers, R, (1996). Prácticas de crianza: Creando programas donde las tradiciones y las prácticas modernas se encuentran. (Sampson, L. y Tenorio, M. Trads.) Coordinator's Notebook CHILDREARING $N^{\circ} 15$, (1994), UNICEF.: Universidad del Valle. http://cognitiva.univalle.edu. co/archivos/grupo \%20cultura/recursos/Pr\%E1cticas\%20de\%20crianza.pdf

García, B., Loredo, J., y Carranza, G. (2008). Análisis de la práctica educativa de los docentes: pensamiento, interacción y reflexión. Revista Electrónica de Investigación Educativa, 2-15. http:// redie.uabc.mx/NumEsp1/contenido-garcialoredocarranza.html

González, M., de Damas, y Vicente, M. Á. G. (2020). La verbalización de las emociones en educación infantil. evaluación de un programa de conciencia emocional. Estudios Sobre Educación, 38, 279-302. doi: http://dx.doi.org/10.15581/004.38.279-302

Hernández, C. (2012). Competencias familiares para el desarrollo infantil en la gestación y primer año de vida. Revista Virtual Universidad Católica del Norte. (37), 93-111. http://www.redalyc. org/articulo.oa?id=194224568006

Hernández Sampieri, R., Fernández, C., y Baptista, P. (2014). Metodología de la investigación. Sexta Edición. México: Mc Graw Hill. 
Linde, A. (2010). Síntesis y valoración de la teoría sobre el desarrollo moral de Lawrence Kohlberg. Ágora. 29(2). ISSN 0211-6642. pp.31-54. http://dspace.usc.es/bitstream/10347/7373/1/pg_032055_agora29-2.pdf

López, È. (2005). La educación emocional en la educación infantil. Revista interuniversitaria de formación del profesorado, (19)3, 153-167. https://dialnet.unirioja.es/descarga/articulo/2126770. pdf

Martínez, M., y García, M. C. (2012). La crianza como objeto de estudio actual desde el modelo transaccional. Revista Latinoamericana de Ciencias Sociales, Niñez y Juventud, 10(1), 169-178. http://www.scielo.org.co/scielo.php?script=sci_arttext\&pid=S1692-715X2012000100010\&ln$\mathrm{g}=$ en\&tlng=es.

Merino, M. (2015). Educar las emociones: Una reflexión sobre los programas de educación emocional en la primera infancia a través de la lente de la Psicología Positiva. (Proyecto de Investigación para optar al título de Psicóloga) Universidad del Rosario. http://repository.urosario.edu.co/ bitstream/handle/10336/10622/1072663735-2015.pdf?sequence=1\&isAllowed=y

Mineducación (2017). ¿Quiénes son los agentes educativos? http://www.mineducacion.gov.co/primerainfancia/1739/article-177854.html

Mineducación (2014). Documento N²0. Sentido de la educación inicial. http://www.mineducacion. gov.co/1621/article-341810.html

Ministerio de Salud. República de Colombia (1993). Resolución Nº 008430 de 1993. https://www. minsalud.gov.co/sites/rid/Lists/BibliotecaDigital/RIDE/DE/DIJ/RESOLUCION-8430-DE-1993. PDF

Moreno, N. (2008). La crianza en grupos familiares contemporáneos y su cambio a través de las trayectorias vitales de hijos e hijas: estudios de caso en la ciudad de Bogotá. (Tesis para optar el título de Magíster en Psicología). Universidad Nacional de Colombia. http://www.bdigital.unal. edu.co/10519/1/13928003.pdf

Narodowski, M., y Snaider, C. (2017). ¿Bebés en las escuelas? Infancias hiperescolarizadas en una cultura prefigurativa. Revista Latinoamericana De Ciencias Sociales, Niñez y Juventud, 15(1), 45-57. doi: http://dx.doi.org.ezproxy.uniminuto.edu/10.11600/1692715x.1510121052016--

Paniagua, G., y Palacios, J. (2005). ¿Es infantil la educación infantil? En: Educación infantil: respuesta educativa a la diversidad. 13-36, Alianza

Quecedo, R., y Castaño, C. (2002). Introducción a la metodología de investigación cualitativa. Revista de Psicodidáctica, (14), 5-39. http://www.redalyc.org/articulo.oa?id=17501402

Ramírez, A., Ferrando, A., y Sainz, M. (2015). ¿Influyen los estilos parentales y la inteligencia emocional de los padres en el desarrollo emocional de sus hijos escolarizados en $2^{\circ}$ ciclo de educación infantil? Acción Psicológica, 12(1), 65-78, Universidad Nacional de Educación a Distancia. http://www.redalyc.org/pdf/3440/344041426007.pdf

Rendón, M. (2016). Consideraciones acerca de las competencias socioemocionales. En: Rendón, M., Cuadros, O., Hernández, B., Monterrosa, D. \& otras (2016). Las competencias socioemocionales en el contexto escolar (pp. 15 - 35). Editorial Universidad de Antioquia.

Rodríguez, C. (2003). Nociones y destrezas básicas sobre el análisis de datos cualitativos. Ponencia en el seminario Internacional: El proceso de Investigación en educación, algunos elementos clave. Santo Domingo (República Dominicana). Facultad Latinoamericana de Ciencias Sociales 
(FLACSO). http://www.ugr.es/ ugr_unt/Material\%20M\%F3dulo\%201/Nociones\%20y\%20destrezas $\% 20 \mathrm{~b} \%$ E1 sicas $\% 20$ sobre $\% 20 \mathrm{el} \% 20$ analisis $\% 20 \mathrm{de} \% 20$ datos $\% 20$ cualitativos.pdf

Sacristán, J. (2003). ¿Cómo hemos creado al menor? Las imágenes de la infancia... En: El alumno como invención (pp. 30-118) Morata, S.L.

Sameroff, A. J., \& Chandler, M. J. (1975). Reproductive risk and the continuum of caretaking casualty. En: F. D. Horowitz, M. Hetherington, S. Scarr-Salapatek, \& G. Siegel (Eds.), Review of child development research, (4), 187-244. Chicago: University of Chicago Press.

Schettini, P., y Cortazzo, I. (2015). Análisis de datos cualitativos en la investigación social: procedimientos y herramientas para la interpretación de información cualitativa. Universidad Nacional De la Plata. Facultad de trabajo social. Libros de cátedra. $1^{\text {a }}$ edición. http://sedici.unlp.edu.ar/ bitstream/handle/10915/49017/Documento_completo.pdf?sequence=1

Secretaría de Educación de Medellín (2016). Lineamientos y estándares para la prestación del servicio de atención integral a la primera infancia. Documento interno no publicado, Buen Comienzo.

Serna, C., Hurtado, L., Mejía, L., y Zapata, P. (2000). El niño y la niña y la crianza humanizada: la interacción afectiva adulto - niño/a, su efecto en la población de 0 - 2 años de edad. Fundación Ximena Rico Llano. (Proyecto de Investigación para optar al título de Licenciada en Educación Preescolar). Universidad de Antioquia. http://200.24.17.68:8080/jspui/bitstream/123456789/1092/1/ doc.pdf

Shaffer, D. (2002). Desarrollo social y de la personalidad. Thompson.

Shapiro, L. E. (2008). La inteligencia emocional de los niños. Una guía para padres y maestros. Grupo Zeta.

Soto, C., y Violante, R. (2005). Enseñar contenidos en el jardín maternal: una forma de compartir la crianza. En: Soto, C., Violante, R., Mayol, M., Moreau, L., Porstein, A., Sarlé, P. y Vasta, L. (Eds.) (2005). En el jardín maternal: Investigaciones, reflexiones y propuestas (pp. 31-65). Paidós.

Soto, C., y Violante, R. (2008). Pedagogía de la crianza: un campo teórico en construcción. Paidós SAICF.

Triana, A., Ávila, L., y Malagón, A. (2010). Patrones de crianza y cuidado de niños y niñas en Boyacá. Revista Latinoamericana de Ciencias Sociales, Niñez y Juventud, Manizales, 8, (2), 93 -945. http://revistaumanizales.cinde.org.co/rlcsnj/index.php/Revista-Latinoamericana/article/view/81

UNICEF (2011). Guía sobre pautas de crianza para niños y niñas de 0 a 5 años de edad. ¿Mucho poquito o nada? http://files.unicef.org/uruguay/spanish/guia_crianza.pdf

UNICEF (2012). Desarrollo emocional: Clave para la primera infancia 0 a 3. Fundación Kaleidos. http://www.unicef.org/ecuador/Desarrollo_emocional_0a3_simples.pdf

Vila, I. (2000). Los nuevos contextos de crianza. Cultura y educación: Revista de teoría, investigación y práctica. (19), 3-22. doi: http://dx.doi.org/10.1174/113564000753837070.

Wadsworth, B. (1991). Teoría de Piaget del desarrollo cognoscitivo y afectivo. Ed. Diana. 